AtoZ: novas práticas em informação e conhecimento www.ser.ufpr.br/atoz

Universidade Federal do Paraná

Setor de Ciências Sociais Aplicadas

Curso de Gestão da Informação

Av. Prefeito Lothário Meissner, 632 - Campus III

Jardim Botânico

Curitiba - PR, Brasil

80210-170

Fone: $+55(41) 3360-4389$

Fax: +55(41)3336-4471

E-mail: revistaatoz@ufpr.br

URL: http://www.ser.ufpr.br/atoz

$\begin{array}{ll}\text { Periodicidade: } & \text { Semestral } \\ \text { ISSN: } & 2237-826 X \\ \text { Diretrizes para autores: } & \text { http://ojs.c3sl.ufpr.br/ojs2/index.php/atoz/about/submissions\#authorGuidelines }\end{array}$

Qualis/Capes

B4 - Interdisciplinar / B5 - Ciências Sociais Aplicadas I / B4 - Engenharias III

\title{
Indexada/registrada em
}

Directory of Open Access Journals (DOAJ); Sumários.org; Google Acadêmico; LivRe! Portal para periódicos de livre acesso na Internet; InfoBCl; Latindex Catálogo

Todo o conteúdo da Revista (incluindo-se instruções, política editorial e modelos) está sob uma licença Creative Commons AtribuiçãoNãoComercial-Compartilhalgual 3.0 Não Adaptada.

Ao serem publicados por esta Revista, os artigos são de livre uso em ambientes educacionais, de pesquisa e não comerciais, com atribuição de autoria obrigatória.

O (C) copyright dos artigos e da entrevista pertence aos respectivos autores/entrevistados com cessão de direitos para a AtoZ no que diz respeito à inclusão do material publicado (revisado por pares/pós-print) em sistemas/ferramentas de indexação, agregadores ou curadores de conteúdo. Os autores têm permissão e são encorajados a depositar seus artigos em páginas pessoais, repositórios e/ou portais institucionais antes (pré-print) e após (pós-print) a publicação na AtoZ. Solicita-se apenas que, quando possível, a referência bibliográfica (incluindo o link/URL do artigo) seja elaborada com base na publicação na AtoZ: novas práticas em informação e conhecimento.

\section{Comitê Editorial}

Dra. Patrícia Zeni Marchiori, Universidade Federal do Paraná (UFPR), Grupo Metodologias para Gestão da Informação UFPR/CNPq, Brasil Msc. Eduardo Michelotti Bettoni, Observatórios Sesi/Senai/IEL, Grupo Metodologias para Gestão da Informação UFPR/CNPq, Brasil Msc. Andre Luiz Appel, Universidade Federal do Rio de Janeiro (UFRJ), Grupo Metodologias para Gestão da Informação UFPR/CNPq, Brasil Dra. Helena Nunes Silva, Universidade Federal do Paraná (UFPR), Grupo Metodologias para Gestão da Informação UFPR/CNPq, Brasil Dra. Denise Fukumi Tsunoda, Universidade Federal do Paraná (UFPR), Grupo Metodologias para Gestão da Informação UFPR/CNPq, Brasil

\section{Conselho Consultivo}

Dra. Ana Esmeralda Carelli, Universidade Estadual de Londrina - UEL, Brasil

Msc. Augusto José Waszczynskyj Antunes das Neves, Universidade Federal do Paraná - UFPR, Brasil

Dra. Avanilde Kemczinski, Universidade do Estado de Santa Catarina - UDESC, Brasil

Dr. Carlos Olavo Quandt, Pontifícia Universidade Católica do Paraná - PUC PR, Brasil

Dra. Cassandra Ribeiro Joye, Instituto Federal de Educação, Ciência e Tecnologia do Ceará - IFCE, Brasil

Dra. Cláudia Regina Z. Bomfá, Universidade Federal de Santa Maria - UFSM, Brasil

Dr. Claudio Cesar de Sá, Universidade do Estado de Santa Catarina - UDESC, Brasil

Dr. Daniel Cebrian Robles, Consultor independente, Espanha

Dra. Deborah Ribeiro Carvalho, Pontifícia Universidade Católica do Parana - PUCPR, Brasil

Dra. Faimara do Rocio Strauhs, Universidade Tecnológica Federal do Paraná - UTFPR, Brasil

Dr. Filiberto Felipe Martínez Arellano, Universidad Nacional Autónoma de México - UNAM, México

Dr. Francisco José Ruiz Rey, Universidad de Málaga - UMA, Espanha

Msc. Frank Coelho de Alcântara, Universidade Positivo - UP, Brasil

Professor lan M. Johnson, The Robert Gordon University - RGU, Reino Unido

Dra. Isabela Gasparini, Universidade do Estado de Santa Catarina - UDESC, Brasil

Dr. Jamerson Viegas Queiroz, Universidade Federal do Rio Grande do Norte - UFRN, Brasil

Dra. Janine Kniess, Universidade do Estado de Santa Catarina - UDESC, Brasil

Dr. José Barata Oliveira, Instituto de Desenvolvimento de Novas Tecnologias - UNINOVA, Portugal

Dr. Juan José Monedero Moya, Universidad de Málaga - UMA, Espanha

Dra. Lucila Pérez Cascante, Universidad Casa Grande - UCG, Equador

Dra. Maria Cristina Vieira de Freitas, Universidade de Coimbra - UC, Portugal

Dra. Maria da Graça de Melo Simões, Universidade de Coimbra - UC, Portugal

Dr. Maria do Carmo Duarte Freitas, Universidade Federal do Paraná, Brasil

Dra. María Gladys Ceretta Soria, Universidad de la República - UdelaR, Uruguai

Dra. Maria Salet Ferreira Novellino, Escola Nacional de Ciências Estatísticas - IBGE, Brasil

Dr. Mauro José Belli, Universidade Federal do Paraná - UFPR, Brasil 


\section{SA.to.ZZ}

Msc. Murilo Artur Araújo da Silveira, Universidade Federal de Pernambuco - UFPE, Brasil

Msc. Victor Marcos Ferracutti, Universidad Nacional del Sur - UNS, Argentina

\section{Editores de Seção - Expediente}

Msc. Andre Luiz Appel, Grupo de Pesquisa UFPR/CNPq - Metodologias para Gestão da Informação, Brasil

Msc. Eduardo Michelotti Bettoni, Grupo de Pesquisa UFPR/CNPq - Metodologias para Gestão da Informação, Brasil

Editores de Seção - Editorial

Dra. Patricia Zeni Marchiori, Universidade Federal do Paraná - UFPR, Brasil

\section{Editores de Seção - Entrevistas}

Msc. Eduardo Michelotti Bettoni, Grupo de Pesquisa UFPR/CNPq - Metodologias para Gestão da Informação, Brasil

\section{Editores de Seção - Artigos}

Dra. Patricia Zeni Marchiori, Universidade Federal do Paraná - UFPR, Brasil

Msc. Eduardo Michelotti Bettoni, Grupo de Pesquisa UFPR/CNPq - Metodologias para Gestão da Informação, Brasil

\section{Editores de Seção - Short Papers}

Dra. Patricia Zeni Marchiori, Universidade Federal do Paraná - UFPR, Brasil

Msc. Andre Luiz Appel, Grupo de Pesquisa UFPR/CNPq - Metodologias para Gestão da Informação, Brasil

\section{Editores de Leiaute}

Msc. Andre Luiz Appel, Grupo de Pesquisa UFPR/CNPq - Metodologias para Gestão da Informação, Brasil

Msc. Eduardo Michelotti Bettoni, Grupo de Pesquisa UFPR/CNPq - Metodologias para Gestão da Informação, Brasil

Marcelo Batista de Carvalho, Bolsista UFPR/TN, Grupo de Pesquisa UFPR/CNPq - Metodologias para Gestão da Informação, Brasil

\section{Apoio técnico}

Biblioteca Digital de Periódicos (BDP), UFPR, Brasil

Capa

Andre Luiz Appel, UFRJ, Brasil

\section{Projeto gráfico}

Grupo de Pesquisa UFPR/CNPq - Metodologias para Gestão da Informação

\section{Avaliadores da edição (v. 4 n. 1)}

Msc. Augusto José Antunes Waszczynskyj das Neves - Universidade Federal do Paraná/Campus Litoral - UFPR

Msc. Ana Greef, Programa de Pós-Graduação em Ciência, Gestão e Tecnologia da Informação - UFPR, Brasil

Msc. Andre Appel, Grupo de Pesquisa UFPR/CNPq - Metodologias para Gestão da Informação, Brasil

Dr. Cícero Ap. Bezerra - Universidade Federal do Paraná - UFPR

Dra. Denise Fukumi Tsunoda - Universidade Federal do Paraná - UFPR

Dra. Faimara do Rocio Strauhs - Universidade Federal Tecnológica do Paraná - UTFPR

Dr. Gregório Varvakis Rados - Universidade Federal de Santa Catarina - UFSC

Dra. Helena de Fátima Nunes Silva - Grupo de Pesquisa UFPR/CNPq - Metodologias para Gestão da Informação, Brasil

Dra. Maria Cristina Freitas, Universidade de Coimbra - UC, Portugal

Msc. Murilo Artur Silveira, Universidade Federal de Pernambuco - UFPE, Brasil

DOI da edição (v. 4 n. 1)

10.5380/atoz/v4i1

AtoZ: Novas Práticas em Informação e Conhecimento. - Vol. 4, n. 1 (jan./jun. 2015)- . Curitiba : Universidade Federal do Paraná, Curso de Gestão da Informação, 2015- .

v.

Semestral.

Publicação online: <http://www.ser.ufpr.br/atoz>

ISSN 2237-826X

1. Comunicação científica - Periódico. 2. Informação - Periódico. 3. Conhecimento -

Periódico.

I. Curso de Gestão da Informação. II. Universidade Federal do Paraná. 